\title{
DINÂMICA DAS CARACTERÍSTICAS QUÍMICAS DE UM SUBSTRATO FLORESTAL EXPOSTO À IRRIGAÇÃO
}

\author{
DYNAMICS OF THE CHEMICAL CHARACTERISTICS OF A FOREST SUBSTRATE EXPOSED TO \\ IRRIGATION
}

\author{
Michael Silveira Thebaldi ${ }^{1}$ Luiz Antonio Lima ${ }^{2}$ Matheus de Figueiredo Braga Colares ${ }^{3}$ \\ Antônio Carlos da Silva ${ }^{4}$ Pedro Luiz Terra Lima ${ }^{5}$
}

\begin{abstract}
RESUMO
A qualidade das mudas florestais produzidas em viveiros está relacionada a diversos fatores, entre eles a irrigação e o substrato utilizado. Ambos possuem como finalidade a produção de mudas de qualidade em um curto período de tempo, mas a irrigação em excesso pode influenciar negativamente no conteúdo de nutrientes do substrato. Assim, este trabalho teve como objetivo avaliar a dinâmica das características químicas de um substrato florestal submetido a sucessivas irrigações em tanques de subirrigação em dois tamanhos de tubetes. Para tal, foram colocados tubetes de 110 e $300 \mathrm{~cm}^{3}$ contendo substrato florestal novo, sem a presença de mudas, em dois tanques de subirrigação com recirculação, um para cada tamanho de tubete, construídos na Universidade Federal de Lavras. Foi montado um Delineamento Inteiramente Casualizado com duas repetições, sendo os níveis do tratamento $0,6,12,18,24$ e 30 irrigações, cinco por dia. As características químicas avaliadas do substrato foram: $\mathrm{pH}$ em água, fósforo, potássio, ferro, zinco, manganês, cobre, cálcio, magnésio, alumínio, boro, enxofre, CTC efetiva, matéria orgânica e condutividade elétrica. Adicionalmente, foram coletadas, antes das irrigações contabilizadas como tratamento, amostras de água para verificação de possíveis alterações na qualidade da água de irrigação reciclada após as sucessivas irrigações. As subirrigações proporcionaram alterações na concentração apenas de potássio no substrato em ambos os tubetes, sendo que a maioria das características químicas avaliadas nos substratos permaneceu inalterada em função das subirrigações.
\end{abstract}

Palavras-chave: viveiro; subirrigação; lixiviação.

\begin{abstract}
The quality of seedlings produced at forest nurseries is related to several factors, including irrigation and the used substrate. Both have as their purposes the production of quality seedlings in a short time period, but over-irrigation can negatively affect the substrate nutrient content. This work aimed to evaluate the chemical characteristics dynamics of a forest substrate subjected to successive irrigation at sub-irrigation tanks in two plastic tubes sizes. To this end, plastic tubes of 110 and $300 \mathrm{~cm}^{3}$ were placed, containing

1 Engenheiro Agrícola, Doutor em Recursos Hídricos em Sistemas Agrícolas, Professor Titular, Setor de Engenharia Ambiental e Sanitária, Centro Universitário de Formiga - MG, Avenida Dr. Arnaldo de Senna 328, Água Vermelha, CEP 35570-000, Formiga (MG), Brasil.msthebaldi@uniformg.edu.br

2 Engenheiro Agrícola, Ph.D., Professor Associado do Departamento de Engenharia, Universidade Federal de Lavras, Campus Universitário, Caixa Postal 3037, CEP 37200-000, Lavras (MG), Brasil. lalima@deg.ufla.br

3 Engenheiro Agrônomo, Doutorando em Recursos Hídricos em Sistemas Agrícolas, Departamento de Engenharia, Núcleo Didático Científico de Engenharia de Água e Solo, Universidade Federal de Lavras, Campus Universitário, Caixa Postal 3037, CEP 37200-000, Lavras (MG), Brasil. matheus-colares@hotmail.com

4 Engenheiro Agrícola, Doutor em Engenharia Agrícola, Departamento de Engenharia, Núcleo Didático Científico de Engenharia de Água e Solo, Universidade Federal de Lavras, Campus Universitário, Caixa Postal 3037, CEP 37200-000, Lavras (MG), Brasil. engcarlinhos@hotmail.com

5 Engenheiro Agrícola, Doutorando em Ciências do Solo, Departamento de Ciência do Solo, Universidade Federal de Lavras, Campus Universitário, Caixa Postal 3037, CEP 37200-000, Lavras (MG), Brasil. pedroterralima@ yahoo.com.br
\end{abstract}

Recebido para publicação em 12/04/2012 e aceito em 4/09/2013 
forest substrate without the presence of new seedlings at two sub-irrigation tanks with recirculation, one for each tube size, built at Universidade Federal de Lavras. It was set up completely randomized design with two replications, being the treatment levels $0,6,12,18,24$ and 30 irrigations, five per day. The substrate chemical characteristics were evaluated: $\mathrm{pH}$, phosphorus, potassium, iron, zinc, manganese, copper, calcium, magnesium, aluminum, boron, sulfur, effective CEC, organic matter and electrical conductivity. Additionally, before the irrigations counted as treatment, water samples were taken to check for possible changes in the quality of irrigation recycled water after successive irrigations. The sub-irrigations provided only changes in the potassium concentration in the substrate in both tubes, and most of the chemical characteristics assessed on the substrate were unchanged as a sub-irrigation function.

Keywords: nursery; sub-irrigation; leaching.

\section{INTRODUÇÃO}

A demanda por produtos de origem florestal aumentou sensivelmente nas últimas décadas, levando a silvicultura a buscar alternativas que pressupõem altas produtividades (BOLFE et al., 2004), como a melhoria da qualidade das mudas produzidas. Contrariamente, a obtenção de padrões de qualidade da muda e o aprimoramento das técnicas de viveiro não tem acompanhado a evolução conseguida em outras fases do reflorestamento (SANTOS et al., 2000). Neste cenário, o uso correto de insumos, como substratos e recipientes; e técnicas utilizadas, como irrigação e fertilização no cultivo em viveiro, fazem-se essenciais.

O substrato para a produção de mudas tem por finalidade garantir o desenvolvimento de uma planta com qualidade, em curto período de tempo, e baixo custo (CUNHA et al., 2006). Fochesato et al. (2006) citam que as características químicas mais importantes são o $\mathrm{pH}$, a capacidade de troca de cátions, a condutividade elétrica e/ou teor total de sais solúveis. Segundo Santos et al. (2000), os problemas mais comuns na produção de mudas referem-se às condições de acidez excessivas do substrato. A acidez pode atuar de maneira direta sobre as plantas, ocasionando injúrias, ou de forma indireta, afetando a disponibilidade de nutrientes, produzindo condições bióticas desfavoráveis à fixação do nitrogênio e à atividade de micorrizas, ou ainda aumentando a infecção por alguns patógenos. Entre os efeitos indiretos, a relação entre a disponibilidade de nutrientes e o valor do $\mathrm{pH}$ apresenta maior importância, especialmente a disponibilidade de nitrogênio, enxofre e potássio diminuída em meio ácido (WALLER e WILSON, 1984).

Outro fator que pode influenciar negativamente no conteúdo de nutrientes do substrato é a irrigação em excesso, que, aliada ao pequeno volume, pode provocar uma elevada taxa de lixiviação de nutrientes e dificultar a manutenção dos nutrientes em níveis adequados, especialmente de nitrogênio (WENDLING e GATTO, 2002). Para produção em viveiros e casas de vegetação, empregam-se maiores taxas de fertilização que em outras culturas (MOLITOR, 1990), assim, a água perdida pode ter uma quantidade significativa de fertilizante não utilizado (JUNTENEN et al., 2002; DUMROESE et al., 2005) e ser uma fonte potencial de poluição da água subsuperficial e superficial (DUMROESE et al., 2006) nas proximidades do viveiro. Portanto, a subirrigação apresenta-se como solução relevante no que se refere ao ganho em eficiência de uso da água. Quando a irrigação é completada, a água não utilizada é drenada de volta ao reservatório para posterior recirculação pelo sistema (STRONG et al., 1997; DUMROESE et al., 2006). Já que o sistema é fechado, dados publicados por Ahmed et al. (2000) revelam uma redução em $86 \%$ de uso da água em subirrigação comparado com irrigação sobrecopa, em culturas alimentares, e a perda de fertilizantes para o solo é, praticamente, eliminada, já que a água lixiviada é recirculada, impedindo que fertilizantes e pesticidas atinjam os lençóis superficiais e profundos. Podese ressaltar ainda a redução no consumo de energia elétrica (CHANSEETIS et al., 2001; TOSHIAKI et al., 2004).

Em vista do exposto, o presente trabalho teve como objetivo avaliar a dinâmica das características químicas de um substrato florestal submetido a sucessivas irrigações em tanques de subirrigação em dois volumes de tubetes. 


\section{MATERIAIS E MÉTODOS}

A subirrigação foi realizada utilizando dois tanques, um para bandejas de tubetes pequenos (110 $\left.\mathrm{cm}^{3}\right)$ e outro para bandejas de tubetes grandes $(300$ $\mathrm{cm}^{3}$ ), com dimensões de $1,65 \mathrm{~m}$ x 2,55 $\mathrm{m}$ e 1,60 $\mathrm{m} \times 2,50 \mathrm{~m}$, respectivamente. Ambos os tanques possuíam $0,2 \mathrm{~m}$ de profundidade e altura total de 1,10 $\mathrm{m}$, construídos de blocos de concreto (dimensões de $0,40 \mathrm{~m} \times 0,20 \mathrm{~m} \times 0,15 \mathrm{~m}$ ), na Universidade Federal de Lavras (UFLA).

Encontram-se na Figura 2 os detalhes dos tanques de subirrigação como: vista lateral (1A), tubulação de entrada de água com hidrômetro (1B), tubulação de saída de água e reservatórios para bombeamento e armazenamento da água drenada (1C) e vista lateral superior do tanque para tubetes pequenos (1D).

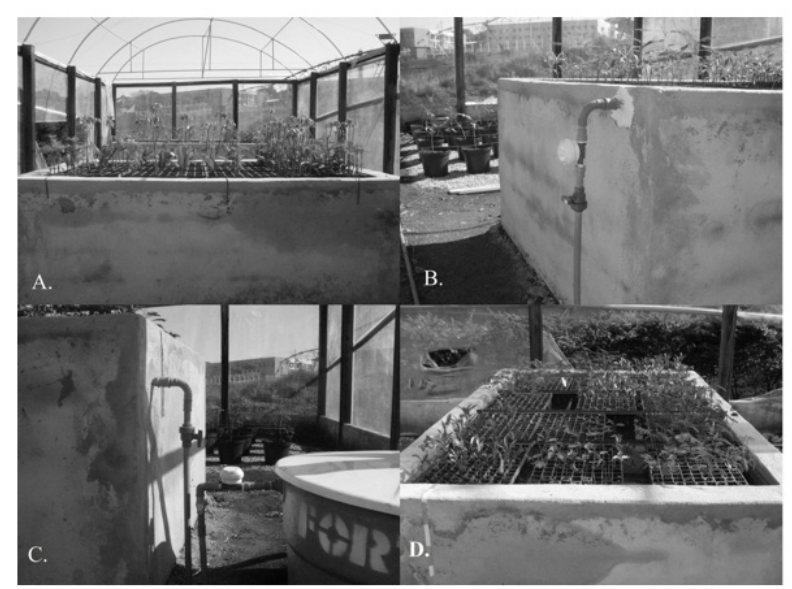

FIGURA 1: Detalhes dos tanques de subirrigação. FIGURE 1: Overviews of sub-irrigation tanks.

O sistema de alimentação dos tanques foi constituído por uma motobomba Schneider modelo $\mathrm{BC}-98 \mathrm{com}$ potência de $0,25 \mathrm{cv}$, que retirava a água de duas caixas d'água de 500 litros em fibra, cada uma destas ligada a um tanque de subirrigação.

A lâmina de água aplicada por tanque foi de $0,05 \mathrm{~m}$ a partir da parte inferior do tubetes. O tempo de irrigação de tubetes grandes era de 0,75 horas e de 0,5 horas para tubetes pequenos, tempos estes necessários para que a frente de umidade vinda por ascensão capilar chegasse à camada superior de substrato. A água de irrigação era bombeada para os tanques era retirada por meio da mesma motobomba utilizada para a alimentação, retornando à sua caixa d'água correspondente.

Os tempos de irrigação representavam umidades de $0,55 \mathrm{~cm}^{3} \mathrm{~cm}^{-3}$ em tubetes grandes e $0,67 \mathrm{~cm}^{3} \mathrm{~cm}^{-3} \mathrm{em}$ tubetes pequenos tendo o substrato $0,38 \mathrm{~cm}^{3} \mathrm{~cm}^{-3}$ de umidade inicial, correspondentes a tensões de 2,34 kPa e 1,50 kPa, respectivamente, em tubetes contendo substrato sem plantas. $\mathrm{O}$ substrato utilizado foi o Tropstrato Florestal ${ }^{\circledR}$ fabricado pela empresa Vida Verde, composto por fibra de coco, vermiculita, carvão vegetal e casca de pinus em proporções não informadas pelo fabricante e a curva de retenção de água deste foi obtida com o software SWRC versão 3.0 da ESALQ/USP, utilizandose o modelo de Van Genuchten (1980), e pode ser visualizada na Figura 2.

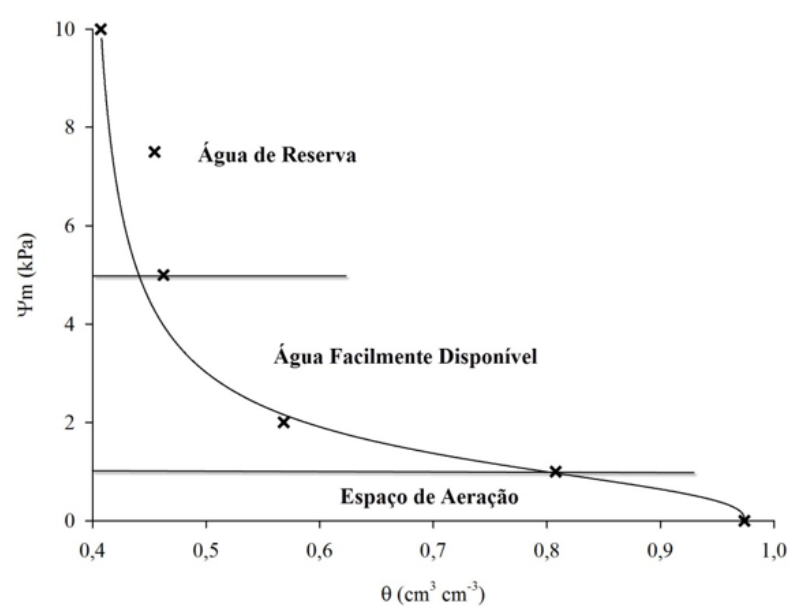

FIGURA 2: Curva de retenção de água do substrato. FIGURE 2: Substrate water retention curve.

Segundo a nomenclatura sugerida por López (2000) e destacada na Figura 2, a porosidade total do substrato utilizado era de $0,974 \mathrm{~cm}^{3} \mathrm{~cm}^{-3}$, Espaço de Aeração de $0,170 \mathrm{~cm}^{3} \mathrm{~cm}^{-3}$, Água Facilmente Disponível de $0,362 \mathrm{~cm}^{3} \mathrm{~cm}^{-3}$, Água de Reserva de $0,034 \mathrm{~cm}^{3} \mathrm{~cm}^{-3}$ e Água Residual de $0,408 \mathrm{~cm}^{3} \mathrm{~cm}^{-3}$.

Para reduzir a perda de substrato nos tubetes no momento da drenagem da água, foram fixados na parte inferior de cada tubete, com auxílio de gomas elásticas, quadrados de aproximadamente $0,07 \mathrm{~m}$ de lado de Tule (manta tipo esponja). Esta medida foi tomada para que não houvesse perda de substrato durante as avaliações, o que poderia ser confundido com uma possível perda de água.

Para avaliar a dinâmica das características químicas do substrato em função do número de subirrigações foram colocados tubetes grandes e pequenos com substrato novo e sem conter plantas em cada tanque de subirrigação. Foi utilizado o Delineamento Inteiramente Casualizado com duas repetições, sendo os níveis do tratamento 0 , 
$6,12,18,24$ e 30 irrigações. Foram realizadas 5 irrigações por dia e quando se acumulava o número de irrigações correspondentes a algum tratamento, retiravam-se amostras de substrato.

As análises químicas do substrato foram realizadas no Laboratório de Fertilidade do Solo do Departamento de Ciências do Solo da UFLA, sendo avaliado: $\mathrm{pH}$ em água, fósforo, potássio, ferro, zinco, manganês, cobre (extrator Mehlich 1), cálcio, magnésio, alumínio (extraídos por cloreto de potássio), boro (extraído por água quente), enxofre (extraído por fosfato monocálcio em ácido acético), matéria orgânica (determinada por meio de sua oxidação pelo $\mathrm{Na}_{2} \mathrm{Cr}_{2} \mathrm{O}_{7} 4 \mathrm{~N}+\mathrm{H}_{2} \mathrm{SO}_{4} 10 \mathrm{~N}$ ), condutividade elétrica e após calculada a capacidade de troca de cátions (efetiva).

Foram coletadas amostras de água para verificação de possíveis alterações na qualidade da água de irrigação após as sucessivas irrigações antes das irrigações contabilizadas como tratamento, sendo avaliados os parâmetros: fósforo, potássio, sódio, cálcio, magnésio, Razão de Adsorção de Sódio (RAS), alumínio, zinco, ferro total, manganês, cobre, boro, enxofre, $\mathrm{pH}$ e condutividade elétrica. As análises da água de irrigação foram realizadas no Laboratório de Fertilidade do Solo do Departamento de Ciências do Solo da UFLA.

A Razão de Adsorção de Sódio (RAS) pode ser calculada pela Equação 1:

$$
\mathrm{RAS}=\frac{\mathrm{Na}^{+}}{\sqrt{\frac{\mathrm{Ca}^{++}+\mathrm{Mg}^{++}}{2}}}(1)
$$

Em que: RAS = Razão de Adsorção de Sódio, meq $\mathrm{L}^{-1} ; \mathrm{Na}^{+}=$concentração do íon sódio, em meq $\mathrm{L}^{-1}$; $\mathrm{Ca}^{++}=$concentração do íon cálcio, em meq $\mathrm{L}^{-1}$; $\mathrm{Mg}^{++}=$concentração do íon magnésio, em meq $\mathrm{L}^{-1}$.

\section{RESULTADOS E DISCUSSÃO}

Os valores dos parâmetros utilizados para avaliar a qualidade da água nas subirrigações são apresentados na Tabela 1 e pelos padrões descritos por Ayers e Westcot (1985), não houve restrição de uso da água de irrigação em nenhuma das irrigações em função de problemas de: salinidade (avaliados pela condutividade elétrica); infiltração (observados através da avaliação em conjunto de condutividade elétrica e RAS) e toxicidade por sódio, também avaliada através da RAS.
Porém, entre 11 e 23 irrigações puderamse observar alterações nos valores de $\mathrm{pH}$, já que através dos padrões de qualidade propostos pelos autores supracitados, a faixa normal para irrigação encontra-se entre 6,5 e 8,4. O aumento do $\mathrm{pH}$ e condutividade elétrica da água de irrigação pode ser explicado pelo aumento da concentração de sais de caráter básico na água de irrigação como potássio, cálcio, magnésio e sódio (Tabela 1).

Montesano et al. (2010) irrigaram tomate em cultivo sem solo em vasos via subirrigação em ciclo fechado e registraram aumento da condutividade elétrica na solução nutritiva utilizada na subirrigação de valores próximos a $3,5 \mathrm{dS} \mathrm{m}^{-1}$ até aproximadamente $5,5 \mathrm{dS} \mathrm{m}^{-1}$ quando a solução nutritiva era totalmente recirculada e concentrações de sódio e potássio aumentado de $500 \mathrm{mg} \mathrm{L}^{-1}$ e 350 mg L-1 a $1000 \mathrm{mg} \mathrm{L}^{-1}$ e $500 \mathrm{mg} \mathrm{L}^{-1}$, respectivamente.

As médias de $\mathrm{pH}$, condutividade elétrica e concentrações de fósforo, cálcio, alumínio, CTCef, matéria orgânica, zinco, cobre, boro e enxofre no substrato em função do número de subirrigações em tubetes grandes podem ser vistas na Tabela $2 \mathrm{e}$ como mostrado nesta, o número de subirrigações não influenciou a concentração no substrato dos parâmetros nela apresentada.

Segundo proposta de classificação apresentada por Gonçalves e Poggiani (1996) para interpretação de características químicas de substratos utilizados na produção de mudas florestais, os valores de fósforo em todas as irrigações podem ser considerados baixos (menores que $200 \mathrm{mg} \mathrm{dm}^{-3}$ ), CTCef encontra-se em nível médio (entre 10 e $20 \mathrm{cmol}_{\mathrm{c}} \mathrm{dm}^{-3}$ ) e o $\mathrm{pH}$ em nível adequado (entre 5,5 e 6,5).

A manutenção das concentrações de matéria orgânica do substrato em tubetes grandes em 30 subirrigações (Tabela 3) mostra não haver degradação destas em função das irrigações o que também reflete na constância também apurada nos níveis de CTCef (Tabela 2). As concentrações de zinco, cobre, boro e enxofre também não sofreram alterações em função das subirrigações.

Sampaio et al. (2008), em avaliação de substrato composto unicamente por fibra de coco, obtiveram concentração de $2,48 \mathrm{dag} \mathrm{dm}^{-3}$ de matéria orgânica, $200 \mathrm{mg} \mathrm{dm}^{-3}$ de enxofre e $0,29 \mathrm{mg} \mathrm{dm}^{-3} \mathrm{de}$ cobre, valores inferiores aos obtidos e $11,76 \mathrm{mg} \mathrm{dm}^{-3}$ de zinco, $\mathrm{pH}$ de 6,6, valores superiores aos obtidos no substrato utilizado, que o fabricante informa ser composto principalmente, mas não unicamente, por fibra de coco. 
TABELA 1: Parâmetros de qualidade da água utilizada nos ciclos de subirrigação.

TABLE 1: Quality parameters of the water used on sub-irrigation cycles.

\begin{tabular}{ccccccc}
\hline \multirow{2}{*}{ Parâmetro } & \multicolumn{7}{c}{ Número de Irrigações } \\
\cline { 2 - 7 } & 0 & 5 & 11 & 17 & 23 & 29 \\
\hline $\mathrm{P}$ & 0,1 & 0,1 & 0,2 & 0,2 & 0,3 & 0,3 \\
$\mathrm{~K}$ & 16,0 & 56,0 & 70,0 & 72,0 & 80,0 & 89,0 \\
$\mathrm{Na}$ & 24,8 & 78,2 & 101,2 & 101,2 & 110,4 & 123,3 \\
$\mathrm{Ca}$ & 9,7 & 13,7 & 15,1 & 13,9 & 14,8 & 15,9 \\
$\mathrm{Mg}$ & 0,5 & 0,6 & 0,8 & 0,8 & 0,9 & 1,0 \\
$\mathrm{RAS}$ & 0,11 & 0,28 & 0,35 & 0,36 & 0,38 & 0,41 \\
$\mathrm{Al}$ & 0,1 & 0,0 & 0,0 & 0,0 & 0,0 & 0,0 \\
$\mathrm{Zn}$ & 0,1 & 0,1 & 0,1 & 0,1 & 0,1 & 0,1 \\
$\mathrm{Fe}$ & 0,1 & 0,1 & 0,1 & 0,1 & 0,1 & 0,1 \\
$\mathrm{Mn}$ & 0,1 & 0,1 & 0,1 & 0,1 & 0,1 & 0,1 \\
$\mathrm{Cu}$ & 0,1 & 0,1 & 0,1 & 0,1 & 0,1 & 0,1 \\
$\mathrm{~B}$ & 0,1 & 0,1 & 0,0 & 0,0 & 0,0 & 0,0 \\
$\mathrm{~S}$ & 5,8 & 10,8 & 9,4 & 18,3 & 18,9 & 13,8 \\
$\mathrm{pH}$ & 7,3 & 8,1 & 9,4 & 9,4 & 9,3 & 8,2 \\
$\mathrm{CE}$ & 0,074 & 0,162 & 0,202 & 0,197 & 0,221 & 0,263 \\
\hline
\end{tabular}

Em que: $\mathrm{P}=$ fósforo $\left(\mathrm{mg} \mathrm{dm}^{-3}\right) ; \mathrm{K}=$ potássio $\left(\mathrm{mg} \mathrm{dm}^{-3}\right) ; \mathrm{Na}=$ sódio $\left(\mathrm{mg} \mathrm{dm}^{-3}\right) ; \mathrm{Ca}=$ cálcio $\left(\mathrm{cmol} \mathrm{dm}^{-3}\right)$; $\mathrm{Mg}=$ magnésio $\left(\mathrm{cmol} \mathrm{dm}^{-3}\right) ; \mathrm{RAS}=$ razão de adsorção de sódio; $\mathrm{Al}=$ alumínio $\left(\mathrm{cmol} \mathrm{dm}^{-3}\right) ; \mathrm{Zn}=$ zinco $\left(\mathrm{mg} \mathrm{dm}^{-3}\right)$; $\mathrm{Fe}=$ ferro $\left(\mathrm{mg} \mathrm{dm}^{-3}\right) ; \mathrm{Mn}=$ manganês $\left(\mathrm{mg} \mathrm{dm}^{-3}\right) ; \mathrm{Cu}=$ cobre $\left(\mathrm{mg} \mathrm{dm}^{-3}\right) ; \mathrm{B}=$ boro $\left(\mathrm{mg} \mathrm{dm}^{-3}\right) ; \mathrm{S}=$ enxofre $\left(\mathrm{mg} \mathrm{dm}^{-3}\right)$; $\mathrm{pH}=$ potencial hidrogeniônico; $\mathrm{CE}=$ condutividade elétrica $\left(\mathrm{dS} \mathrm{m}^{-1}\right)$.

TABELA 2: Médias de $\mathrm{pH}$, condutividade elétrica e concentrações de fósforo, cálcio, alumínio, CTCef, matéria orgânica, zinco, cobre, boro e enxofre no substrato em função do número de subirrigações em tubetes grandes.

TABLE 2: Average values of $\mathrm{pH}$, electric conductivity, phosphorus, calcium, aluminum, CEC, organic matter, zinc, copper, boron and sulfur on substrate in function of the sub-irrigation number in large tubes.

\begin{tabular}{cccccccccccc}
\hline \multirow{2}{*}{ Irrigações } & \multicolumn{10}{c}{ Parâmetros } \\
\cline { 2 - 11 } & $\mathrm{CE}$ & $\mathrm{pH}$ & $\mathrm{P}$ & $\mathrm{Ca}$ & $\mathrm{Al}$ & $\mathrm{CTCef}$ & $\mathrm{MO}$ & $\mathrm{Zn}$ & $\mathrm{Cu}$ & $\mathrm{B}$ & $\mathrm{S}$ \\
\hline 0 & $1,39 \mathrm{a}$ & $5,6 \mathrm{a}$ & $64,4 \mathrm{a}$ & $10,8 \mathrm{a}$ & $0,2 \mathrm{a}$ & $18,5 \mathrm{a}$ & $17,9 \mathrm{a}$ & $5,9 \mathrm{a}$ & $1,0 \mathrm{a}$ & $1,0 \mathrm{a}$ & $204,6 \mathrm{a}$ \\
6 & $1,85 \mathrm{a}$ & $5,7 \mathrm{a}$ & $71,9 \mathrm{a}$ & $9,1 \mathrm{a}$ & $0,2 \mathrm{a}$ & $15,7 \mathrm{a}$ & $16,6 \mathrm{a}$ & $3,0 \mathrm{a}$ & $0,9 \mathrm{a}$ & $0,9 \mathrm{a}$ & $183,1 \mathrm{a}$ \\
12 & $1,74 \mathrm{a}$ & $5,8 \mathrm{a}$ & $71,7 \mathrm{a}$ & $12,3 \mathrm{a}$ & $0,2 \mathrm{a}$ & $20,0 \mathrm{a}$ & $16,3 \mathrm{a}$ & $2,7 \mathrm{a}$ & $0,7 \mathrm{a}$ & $0,7 \mathrm{a}$ & $253,8 \mathrm{a}$ \\
18 & $1,83 \mathrm{a}$ & $5,8 \mathrm{a}$ & $79,6 \mathrm{a}$ & $8,6 \mathrm{a}$ & $0,1 \mathrm{a}$ & $15,0 \mathrm{a}$ & $16,9 \mathrm{a}$ & $2,8 \mathrm{a}$ & $0,8 \mathrm{a}$ & $0,7 \mathrm{a}$ & $264,5 \mathrm{a}$ \\
24 & $1,63 \mathrm{a}$ & $5,8 \mathrm{a}$ & $77,5 \mathrm{a}$ & $8,4 \mathrm{a}$ & $0,1 \mathrm{a}$ & $14,6 \mathrm{a}$ & $14,6 \mathrm{a}$ & $2,7 \mathrm{a}$ & $0,7 \mathrm{a}$ & $0,8 \mathrm{a}$ & $234,2 \mathrm{a}$ \\
30 & $1,48 \mathrm{a}$ & $5,9 \mathrm{a}$ & $69,3 \mathrm{a}$ & $9,7 \mathrm{a}$ & $0,1 \mathrm{a}$ & $16,0 \mathrm{a}$ & $13,8 \mathrm{a}$ & $2,5 \mathrm{a}$ & $0,8 \mathrm{a}$ & $0,7 \mathrm{a}$ & $301,7 \mathrm{a}$ \\
\hline
\end{tabular}

Em que: Médias seguidas de mesma letra na vertical não diferem entre si. $\mathrm{CE}=$ condutividade elétrica $\left(\mathrm{dS} \mathrm{m}^{-1}\right)$; $\mathrm{pH}=$ potencial hidrogeniônico; $\mathrm{P}=$ fósforo $\left(\mathrm{mg} \mathrm{dm}^{-3}\right) ; \mathrm{Ca}=$ cálcio $\left(\mathrm{cmol} \mathrm{dm}^{-3}\right) ; \mathrm{Al}=$ alumínio $\left(\mathrm{cmol} \mathrm{dm}^{-3}\right)$; CTCef $=$ capacidade de troca catiônica $\left(\mathrm{cmol}_{\mathrm{c}} \mathrm{dm}^{-3}\right) ; \mathrm{MO}=$ matéria orgânica $\left(\right.$ dag kg $\left.^{-1}\right) ; \mathrm{Zn}=$ zinco $\left(\mathrm{mg} \mathrm{dm}^{-3}\right)$; $\mathrm{Cu}=$ cobre $\left(\mathrm{mg} \mathrm{dm}^{-3}\right) ; \mathrm{B}=$ boro $\left(\mathrm{mg} \mathrm{dm}^{-3}\right) ; \mathrm{S}=$ enxofre $\left(\mathrm{mg} \mathrm{dm}^{-3}\right)$.

O número de subirrigações causou redução significativa na concentração de potássio no substrato em tubetes grandes, que pode ser descrita por uma equação linear apresentada na Figura 3 com coeficiente de determinação $\left(\mathrm{R}^{2}\right)$ de $89,07 \%$.
Esta redução significativa pode ser explicada pela alta mobilidade do potássio no substrato, tendo a concentração deste também aumentado na água de irrigação reciclada (Tabela 1). A concentração de potássio inicial do substrato já se encontrava 
em níveis inferiores aos citados por Gonçalves e Poggiani (1996) e com a redução, faz-se mais necessária a adubação de potássio para pleno desenvolvimento das mudas.

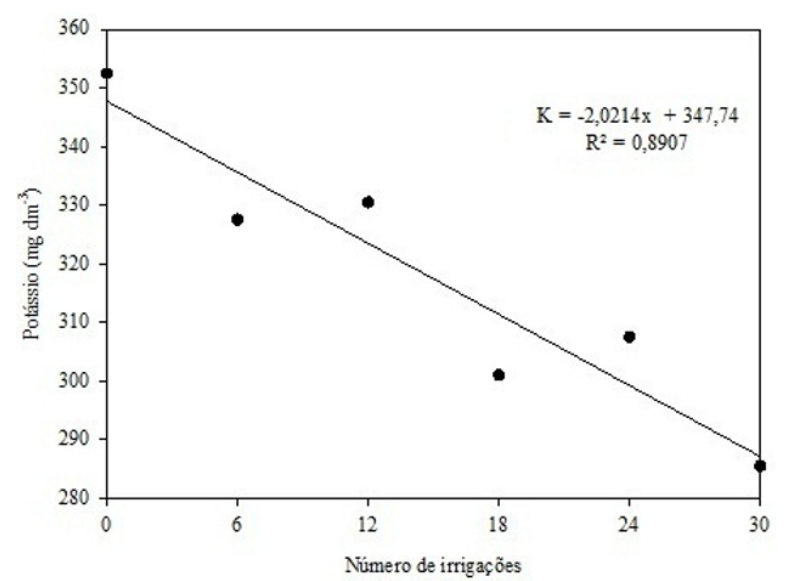

FIGURA 3: Concentração de potássio no substrato em função do número de subirrigações realizadas em tubetes grandes.

FIGURE 3: Potassium concentration on substrate in function of the sub-irrigation numbers in large tubes.

Rozane et al. (2007) avaliaram em substrato composto por casca de Pinus e vermiculita concentração de $67,9 \mathrm{mg} \mathrm{dm}^{-3}$ de potássio, valor inferior ao obtido para o substrato estudado, mesmo após a trigésima irrigação. A resposta da concentração de magnésio no substrato em função do número de subirrigações seguiu uma função quadrática, mostrada na Figura 4, com coeficiente de determinação de $88,73 \%$. A concentração mínima estimada pela equação é de $5,29 \mathrm{cmol} \mathrm{dm}^{-3}$, equivalente a aproximadamente 20 irrigações, nível de concentração deste elemento considerado médio por Gonçalves e Poggiani (1996).

Mesmo sendo obtida uma função quadrática, observa-se uma estabilização dos valores observados a partir de 6 irrigações. A concentração mínima estimada é superior à concentração obtida por Rozane et al. (2007) em substrato de casca de Pinus e vermiculita de $0,245 \mathrm{cmol} \mathrm{dm}^{-3}$ e inferior à obtida por Maeda et al. (2007), na mistura de Plantmax Floresta ${ }^{\circledR}$ com casca de Pinus parcialmente decomposta.

Assim como o ocorrido com as concentrações de potássio em tubetes grandes, o efeito do número de subirrigações sobre a

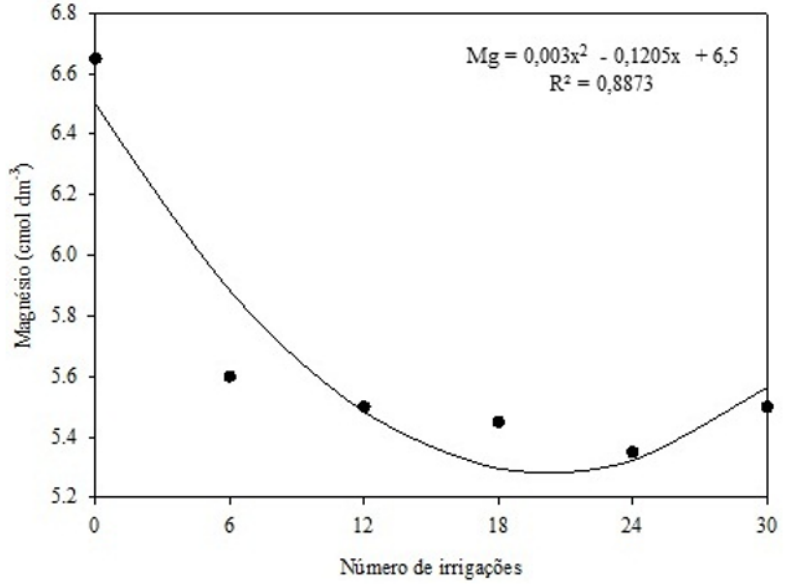

FIGURA 4: Concentração de magnésio no substrato em função do número de subirrigações realizadas em tubetes grandes.

FIGURE 4: Magnesium concentration on substrate due to the number of sub-irrigations in large tubes.

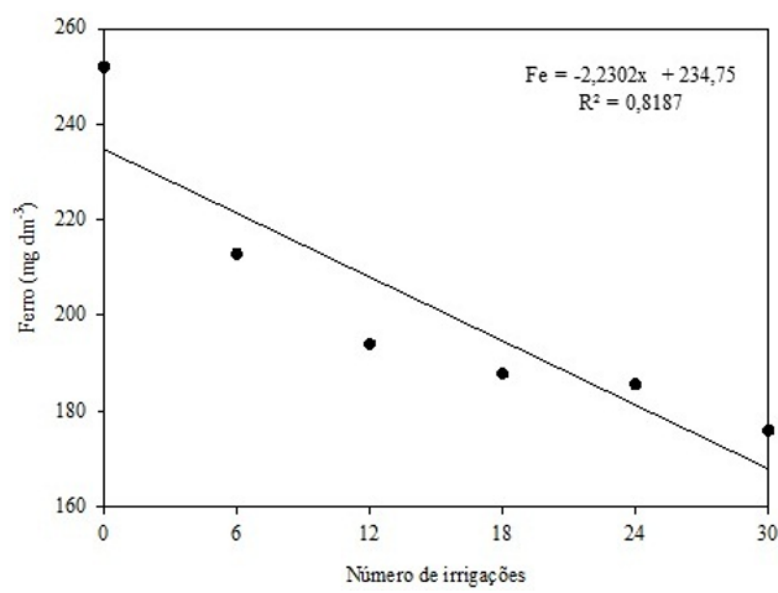

FIGURA 5: Concentração de ferro no substrato em função do número de subirrigações realizadas em tubetes grandes.

FIGURE 5: Iron concentration on substrate due to the number of sub-irrigations in large tubes.

concentração de ferro seguiu um modelo linear, porém, com $\mathrm{R}^{2}$ de $81,87 \%$ (Figura 5).

As subirrigações também causaram redução do ferro contido no substrato, porém, não causaram incremento na concentração deste elemento na água de irrigação (Tabela 1). As concentrações de ferro obtidas, mesmo após a redução ocasionada pelas 30 irrigações, foram superiores às encontradas por Rozane et al. (2007) em substrato de casca de Pinus e vermiculita de $0,2 \mathrm{mg} \mathrm{dm}^{-3}$. 
A estimativa da concentração de manganês em função da subirrigação pode ser determinada por uma função cúbica com $\mathrm{R}^{2}$ de $98,14 \%$ (Figura 6). Assim como ocorreu com o ferro, a alteração nas concentrações de manganês no substrato não refletiram sobre a concentração deste elemento na água de irrigação, sendo as quantidades encontradas superiores às avaliadas por Rozane et al. (2007) em substrato de casca de Pinus e vermiculita (1,3 mg $\left.\mathrm{dm}^{-3}\right)$.

Assim como em tubetes grandes, as subirrigações não causaram alterações significativas nas concentrações de fósforo, CTCef, matéria orgânica, alumínio e no valor de $\mathrm{pH}$ em tubetes pequenos (Tabela 3), mesmo com o aumento do $\mathrm{pH}$, e concentrações de cálcio e magnésio na água de irrigação (Tabela 1). $\mathrm{O}$ aumento da concentração de cálcio e do $\mathrm{pH}$ da água de irrigação não acompanhado do aumento dos valores destes parâmetros nos substratos pode ser devido ao material de revestimento dos tanques de subirrigação, argamassa, material conhecidamente calcário.

As concentrações de fósforo tanto na água de irrigação (Tabela 1) como no substrato em tubetes grandes (Tabela 2) e nos tubetes pequenos não se alteraram significativamente, graças à imobilidade deste nutriente (WENDLING e GATTO, 2002).

A CTCef está diretamente relacionada à disponibilidade de cátions e à redução nas perdas por lixiviação, uma vez que quanto maior a CTCef,

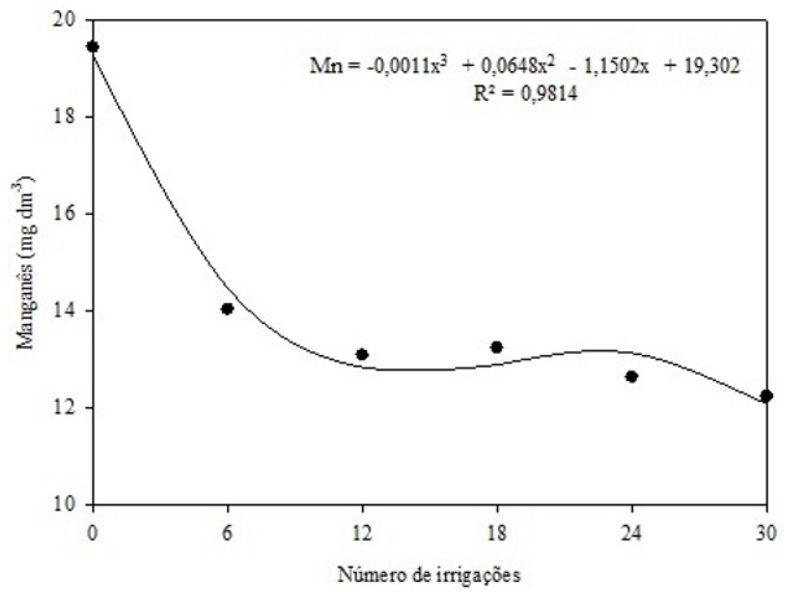

FIGURA6: Concentração de manganês no substrato em função do número de subirrigações realizadas em tubetes grandes.

FIGURE 6: Manganese concentration on substrate due to the numbers of sub-irrigations in large tubes. maior será a retenção de cátions adsorvidos, especialmente em cultivos nos quais a irrigação é frequente (FERMINO, 1996). Valores de 20 $\mathrm{cmol}_{\mathrm{c}} \mathrm{dm}^{-3}$ são recomendados por Martinez (2002), parcialmente atendidas nos valores mostrados nas Tabelas 3 e 4 . Essa parcialidade no atendimento dos requisitos de CTCef citados por esses autores pode explicar o aumento da concentração principalmente de sódio e potássio na água de irrigação e potássio no substrato em tubetes grandes (Figura 3).

As médias dos valores de condutividade elétrica, zinco, ferro, manganês, cobre, boro e enxofre no substrato em função das subirrigações em tubetes pequenos são mostradas na Tabela 4 e, diferentemente do ocorrido em tubetes grandes, em tubetes pequenos, as concentrações de ferro e manganês não sofreram alterações significativas em função das subirrigações.

Os valores de condutividade elétrica do substrato, em tubetes grandes (Tabela 2 ) e pequenos (Tabela 4) não sofreram alteração em função do número de subirrigações.

Sampaio et al. (2008) avaliaram as características químicas de substrato composto unicamente de fibra de coco, obtiveram valor de $2,18 \mathrm{dS} \mathrm{m}^{-1}$ para condutividade elétrica. Bumgarner et al. (2008) obtiveram, utilizando subirrigação no cultivo de mudas de Quercus rubra, valores próximos de $5 \mathrm{dS} \mathrm{m}^{-1}$ no topo, e $1 \mathrm{dS} \mathrm{m}^{-1}$ no meio e base em recipiente de $0,25 \mathrm{~m}$ de altura.

Diferentemente do comportamento da

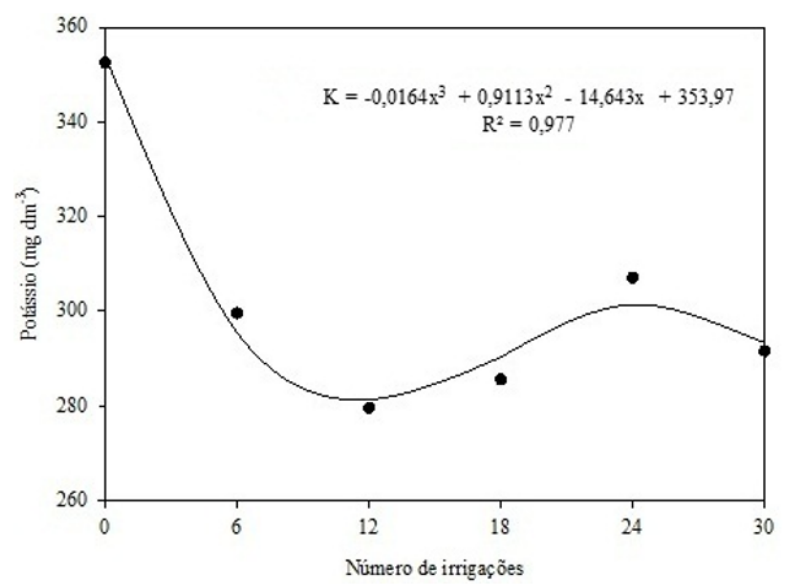

FIGURA 7: Concentração de potássio no substrato em função do número de subirrigações realizadas em tubetes pequenos.

FIGURE 7: Potassium concentration on substrate due to the number of sub-irrigations in small tubes. 
TABELA 3: Médias de pH e das concentrações de fósforo, cálcio, magnésio, CTCef, matéria orgânica e alumínio no substrato em função das subirrigações em tubetes pequenos.

TABLE 3: Average values of $\mathrm{pH}$, phosphorus, calcium, magnesium, CEC, organic matter and aluminum concentrations on substrate due to the sub-irrigations in small tubes.

\begin{tabular}{cccccccc}
\hline \multirow{2}{*}{ Irrigações } & \multicolumn{7}{c}{ Parâmetros } \\
\cline { 2 - 8 } & $\mathrm{pH}$ & $\mathrm{P}$ & $\mathrm{Ca}$ & $\mathrm{Mg}$ & $\mathrm{CTCef}$ & $\mathrm{MO}$ & $\mathrm{Al}$ \\
\hline 0 & $5,6 \mathrm{a}$ & $64,4 \mathrm{a}$ & $10,8 \mathrm{a}$ & $6,7 \mathrm{a}$ & $18,5 \mathrm{a}$ & $17,9 \mathrm{a}$ & $0,2 \mathrm{a}$ \\
6 & $5,9 \mathrm{a}$ & $79,7 \mathrm{a}$ & $16,4 \mathrm{a}$ & $6,4 \mathrm{a}$ & $23,7 \mathrm{a}$ & $15,3 \mathrm{a}$ & $0,1 \mathrm{a}$ \\
12 & $5,9 \mathrm{a}$ & $64,1 \mathrm{a}$ & $8,0 \mathrm{a}$ & $5,0 \mathrm{a}$ & $13,8 \mathrm{a}$ & $14,6 \mathrm{a}$ & $0,1 \mathrm{a}$ \\
18 & $6,0 \mathrm{a}$ & $64,2 \mathrm{a}$ & $8,5 \mathrm{a}$ & $4,9 \mathrm{a}$ & $14,3 \mathrm{a}$ & $15,8 \mathrm{a}$ & $0,1 \mathrm{a}$ \\
24 & $5,9 \mathrm{a}$ & $79,6 \mathrm{a}$ & $8,4 \mathrm{a}$ & $5,1 \mathrm{a}$ & $14,4 \mathrm{a}$ & $13,5 \mathrm{a}$ & $0,1 \mathrm{a}$ \\
30 & $5,9 \mathrm{a}$ & $77,7 \mathrm{a}$ & $9,7 \mathrm{a}$ & $5,8 \mathrm{a}$ & $16,3 \mathrm{a}$ & $14,0 \mathrm{a}$ & $0,1 \mathrm{a}$ \\
\hline
\end{tabular}

Em que: Médias seguidas de mesma letra na vertical não diferem entre si. $\mathrm{P}=$ fósforo $\left(\mathrm{mg} \mathrm{dm}^{-3}\right) ; \mathrm{Ca}=$ cálcio $\left(\mathrm{cmol} \mathrm{dm}{ }^{-3}\right) ; \mathrm{Mg}=$ magnésio $\left(\mathrm{cmol} \mathrm{dm}^{-3}\right) ; \mathrm{t}=$ capacidade de troca catiônica efetiva $\left(\mathrm{cmolc} \mathrm{dm}^{-3}\right) ; \mathrm{MO}=$ matéria orgânica $\left(\mathrm{dag} \mathrm{kg}^{-1}\right) ; \mathrm{Al}=$ alumínio $\left(\mathrm{cmol} \mathrm{dm}^{-3}\right)$.

TABELA 4: Médias de condutividade elétrica e das concentrações de zinco, ferro, manganês, cobre, boro e enxofre no substrato em função das subirrigações em tubetes pequenos.

TABLE 4: Average values electric conductivity and zinc, iron, manganese, copper, boron and sulfer concentrations on substrate due to the numbers of sub-irrigations in small tubes.

\begin{tabular}{cccccccc}
\hline \multirow{2}{*}{ Irrigações } & \multicolumn{7}{c}{ Parâmetros } \\
\cline { 2 - 7 } & $\mathrm{CE}$ & $\mathrm{Zn}$ & $\mathrm{Fe}$ & $\mathrm{Mn}$ & $\mathrm{Cu}$ & $\mathrm{B}$ & $\mathrm{S}$ \\
\hline 0 & $1,39 \mathrm{a}$ & $5,9 \mathrm{a}$ & $251,9 \mathrm{a}$ & $19,5 \mathrm{a}$ & $1,0 \mathrm{a}$ & $1,0 \mathrm{a}$ & $204,6 \mathrm{a}$ \\
6 & $1,30 \mathrm{a}$ & $3,2 \mathrm{a}$ & $218,1 \mathrm{a}$ & $14,8 \mathrm{a}$ & $0,8 \mathrm{a}$ & $0,9 \mathrm{a}$ & $272,3 \mathrm{a}$ \\
12 & $1,36 \mathrm{a}$ & $2,5 \mathrm{a}$ & $185,5 \mathrm{a}$ & $13,6 \mathrm{a}$ & $0,8 \mathrm{a}$ & $0,7 \mathrm{a}$ & $284,6 \mathrm{a}$ \\
18 & $1,20 \mathrm{a}$ & $2,6 \mathrm{a}$ & $193,3 \mathrm{a}$ & $13,5 \mathrm{a}$ & $0,8 \mathrm{a}$ & $0,6 \mathrm{a}$ & $268,6 \mathrm{a}$ \\
24 & $1,26 \mathrm{a}$ & $2,8 \mathrm{a}$ & $197,1 \mathrm{a}$ & $14,6 \mathrm{a}$ & $0,9 \mathrm{a}$ & $0,7 \mathrm{a}$ & $197,5 \mathrm{a}$ \\
30 & $1,13 \mathrm{a}$ & $2,8 \mathrm{a}$ & $226,1 \mathrm{a}$ & $15,6 \mathrm{a}$ & $1,0 \mathrm{a}$ & $0,5 \mathrm{a}$ & $261,1 \mathrm{a}$ \\
\hline
\end{tabular}

Em que: Médias seguidas de mesma letra na vertical não diferem entre si. $\mathrm{CE}=$ condutividade elétrica $\left(\mathrm{dS} \mathrm{m}^{-1}\right) ; \mathrm{Zn}=$ zinco $\left(\mathrm{mg} \mathrm{dm}^{-3}\right) ; \mathrm{Fe}=$ ferro $\left(\mathrm{mg} \mathrm{dm}^{-3}\right) ; \mathrm{Mn}=$ manganês $\left(\mathrm{mg} \mathrm{dm}^{-3}\right) ; \mathrm{Cu}=$ cobre $\left(\mathrm{mg} \mathrm{dm}^{-3}\right) ; \mathrm{B}=$ boro $\left(\mathrm{mg} \mathrm{dm}^{-3}\right) ; \mathrm{S}=$ enxofre $\left(\mathrm{mg} \mathrm{dm}^{-3}\right)$.

concentração de potássio no substrato em tubetes grandes em função das subirrigações (Figura 3) em tubetes pequenos, a estimativa da concentração deste elemento no substrato pode ser explicado por um polinômio de terceiro grau, com coeficiente de determinação de $97,70 \%$, ocorrendo um aumento da concentração após 12 irrigações e voltando a diminuir depois de 24 irrigações (Figura 7). Porém, uma queda acentuada da concentração de potássio só é vista entre o início e seis irrigações, comportamento também apresentado nas Figuras 4 e 6 .

Segundo Resende et al. (1999), espécies pioneiras, em geral, têm seu potencial de crescimento mais restringido quando se desenvolvem em solos pouco férteis, mostrando-se bastante responsivas à fertilização, ao passo que, com o avanço do grupo sucessional, o estímulo ao crescimento proporcionado pela adubação é menos pronunciado e algumas vezes inexistente; tendência, em parte, atribuída ao crescimento mais lento, característico das espécies clímax.

\section{CONCLUSÕES}

As subirrigações proporcionaram alterações na concentração apenas de potássio no substrato em ambos os tubetes. 
As concentrações de magnésio e manganês em tubetes grandes e potássio em tubetes pequenos reduziram até seis irrigações, depois se mantiveram praticamente constantes no substrato.

As concentrações de potássio e ferro em tubetes grandes decresceram linearmente com as irrigações.

Os valores de $\mathrm{pH}, \mathrm{CE}$ e concentrações de fósforo, cálcio, alumínio, CTCef, matéria orgânica, zinco, cobre, boro e enxofre não se alteraram em função das subirrigações em ambos os tubetes.

\section{REFERÊNCIAS BIBLIOGRÁFICAS}

AHMED, A. K.; CRESSWELL, G. C.; HAIGH, A. M. Comparison of sub-irrigation and overhead irrigation of tomato and lettuce seedlings. Journal of Horticultural Science and Biotechnology, Ashford, v. 75, p. $350-354,2000$.

AYERS, R. S.; WESTCOST, D. W. Water quality for agriculture. Rome: $\mathrm{FAO}, 1985.174 \mathrm{p}$.

BOLFE, E. L. et al. Avaliação da classificação digital de povoamentos florestais em imagens de satélite através de índices de Acurácia. Revista Árvore, Viçosa, v. 28, n. 1, p. 85-90, 2004.

BUMGARNER, M. L. et al. Subirrigation of Quercusrubra seedlings: nursery stock quality, media chemistry, and early field performance. Hortscience, Palo Alto, v. 43, n. 7, p. 2179-2185, 2008.

CHANSEETIS, C. et al. Application of capillary hydroponic system to the lettuce growing under tropical climate conditions. Acta Horticulturae, Leuven, v. 548, p. 401-407, 2001.

CUNHA, A. M. et al. Efeito de diferentes substratos sobre o desenvolvimento de mudas de Acacia sp. Revista Árvore, Viçosa, v. 30, n. 2, p. 207-214, 2006.

DUMROESE, R. K. et al. Exponential fertilization of Pinusmonticola seedlings: nutrient uptake efficiency, leaching fractions, and early outplanting performance. Canadian Journal of Forest Research, Ottawa, v. 35, p. 2961-2967, 2005.

DUMROESE, R. K. et al. Subirrigation reduces water use, nitrogen loss, and moss growth in a container nursery. Native Plants Journal, Madison, v. 7, n. 3, p. 253-261, 2006.

FERMINO, M. H. Aproveitamento de resíduos industriais e agrícolas como alternativas de substratos hortícolas. 1996. 90 f. Dissertação (Mestrado em Fitotecnia) - Universidade Federal do Rio Grande do Sul, Porto Alegre, 1996.
FOCHESATO, M. L. et al. Produção de mudas cítricas em diferentes porta-enxertos e substratos comerciais.Ciência Rural, Santa Maria, v. 36, n. 5, p. 1397-1403, 2006.

GONÇALVES, J. L. M.; POGGIANI, F. Substratos para produção de mudas florestais. In: CONCRESSO LATINO AMERICANO DE CIÊNCIA DO SOLO, 13., 1996, Águas de Lindóia. Anais... Águas de Lindóia: USP-ESALQ, 1996. 1 CD ROM.

JUNTENEN, M. L. et al. Ground water quality: leaching of nitrogen and phosphorus during production of forest seedlings in containers. Journal of Environmental Quality, Madison, v. 31, p. 1868-1874, 2002.

LÓPEZ, C. C. Fertirrigacion cultivos horticolas y ornamentales.2. ed. Madrid: Mundi-Prensa, 2000. $475 \mathrm{p}$.

MAEDA, S. et al. Caracterização de substratos para produção de mudas de espécies florestais elaborados a partir de resíduos orgânicos. Pesquisa Florestal Brasileira, Colombo, n. 54, p. 97-104, 2007.

MARTINEZ, P. F. Manejo de substratos para horticultura. In: FURLANI, A. M. C. Caracterização, manejo e qualidade de substratos para produção de plantas.Campinas: IAC, 2002. p. 53-73.

MOLITOR, H. The european perspective with emphasis on subirrigation and recirculation of water and nutrients. Acta Horticulturae, Leuven, v. 272, p. $165-174,1990$.

MONTESANO, F. et al. Closed cycle subirrigation with low concentration nutrient solution can be used for soilless tomato production in saline conditions. Scientia Horticulturae, Scottsville, v. 124, n. 3, p. 338-344, 2010.

RESENDE, A. V. et al. Crescimento inicial de espécies florestais de diferentes grupos sucessionais em resposta a doses de fósforo. Pesquisa Agropecuária Brasileira, Brasília, v. 34, n. 11, p. 2071-2081, 1999.

ROZANE, D. E. et al. Nitrogênio, fósforo e potássio afetando a nutrição e produção de porta-enxerto de limoeiro citrumeloswingle.Revista Ceres, Viçosa, v. 54, n. 315, p. 422-429, 2007.

SAMPAIO, R. A. et al. Produção de mudas de tomateiro em substratos contendo fibra de coco e pó de rocha. Produção de mudas de tomateiro em substratos contendo fibra de coco e pó de rocha. Horticultura Brasileira, Campinas, v. 26, p. 499-503, 2008.

SANTOS, C. B. et al. Efeito do volume de tubetes e tipos de substratos na qualidade de mudas de 
Cryptomeriajaponica (L.F.) D. Don. Ciência Florestal, Santa Maria, v. 10, n. 2, p. 1-15, 2000.

STRONG, S. S. et al. Cultivar and spacing effects on transmission of Phytophthoraparasitica in an ebb-and-flow subirrigation system. Plant Disease, Davis, v. 81, n. 1, p. 89-95, 1997.

TOSHIAKI, A. M. et al. Spatial distribution of ions in groundwater under agricultural land. Journal of Irrigation and Drainage Engineering, Newark, v. 130, n. 6, p. 468, 2004.
VAN GENUCHTEN, M. T. A closed-form equation for predicting the hydraulic conductivity of unsaturated soils.Soil Science Society of America Journal, Madison, v. 44, p. 892-898, 1980.

WENDLING, I.; GATTO, A. Substratos, adubação e irrigação na produção de mudas. Viçosa: Aprenda Fácil, 2002. 165 p.

WALLER, P. L.; WILSON, F. N. Evaluation of growing media for consumer use. Acta Horticulturae, Leuven, n. 150, p. 51-58, 1984. 